\title{
Plant-Based Milks: Hemp ${ }^{1}$
}

\section{Sarah Curl, Daniela Rivero-Mendoza, and Wendy J. Dahl²}

Hemp milk is a plant-based milk growing in popularity. Commercial hemp seed, used in the production of hemp milk, contains only trace amounts of the compound tetrahydrocannabinol (THC), much too low to produce any psychoactive effects from consuming the milk (RodriguezLeyva et al. 2010). This publication describes the composition of hemp milk and the potential health benefits and risks.

\section{How is hemp milk made?}

Commercial hemp milk is made by combining the hemp seed with water (Wang et al. 2018). First, the hemp seeds are mixed with hot water; they swell and soften as they absorb water (Mitchell et al. 2008). This hot slurry of hemp seeds and water is then wet-milled until the desired consistency is reached (Mitchell et al. 2008). The filtered slurry is homogenized for stability and then cooled to stop oxidation of the oils and vitamins (Mitchell et al. 2008). Water, emulsifiers, stabilizers, sweeteners, and flavorings are added to customize the hemp milk (Mitchell et al. 2008). Finally, aseptic processing, such as ultra-high temperature (UHT) treatment, is carried out to extend the product shelf life (Mitchell et al. 2008).

\section{How does the nutrient profile of hemp milk compare to cow's milk?}

The nutrient composition of hemp milk and cow's milk is shown in Table 1. In this comparison, the hemp milk is unsweetened, unflavored, and fortified with calcium.
A serving of unflavored and unsweetened hemp milk provides about 60 calories, less than a serving of cow's milk. Hemp milk provides about 3 grams (g) of protein per serving, whereas cow's milk provides $8 \mathrm{~g}$. Hemp milk is higher in monounsaturated and polyunsaturated fats than low-fat and fat-free cow's milk (USDA n.d.). A serving of unsweetened hemp milk contains no carbohydrate, whereas sweetened varieties may contain more than 20 g per serving, most of which is added sugar (Pacific Foods n.d.-a). In comparison, cow's milk provides $12 \mathrm{~g}$ of carbohydrate from the naturally occurring sugar lactose. A cup of unsweetened hemp milk provides less potassium, but similar amounts of sodium and calcium (when fortified) as cow's milk. Some hemp milks are also fortified with vitamins A, D, B2, and B12 (USDA n.d.). It is important to check the label for the nutrition facts panel or the ingredient list to determine if the hemp milk is fortified.

\section{What are the potential health benefits of hemp milk?}

Hemp seeds are one of the few plant-based foods that have adequate levels of all the essential amino acids to be considered a complete protein (House et al. 2010). Also, hemp seeds are rich in omega-3 fats (USDA n.d.); higher levels of omega-3 fats are associated with human health (Casula et al. 2020). Very little research has specifically examined other health benefits of consuming hemp milk. In a small study, hempseed oil was shown to improve atopic dermatitis (Callaway et al. 2005). This is thought to be due

1. This document is FSHN20-52, one of a series of the Food Science and Human Nutrition Department, UF/IFAS Extension. Original publication date October 2020. Visit the EDIS website at https://edis.ifas.ufl.edu for the currently supported version of this publication.

2. Sarah Curl, former graduate student, Food Science and Human Nutrition Department; Daniela Rivero-Mendoza, Extension and research coordinator; and Wendy J. Dahl, associate professor, Food Science and Human Nutrition Department; UF/IFAS Extension, Gainesville, FL 32611.

The Institute of Food and Agricultural Sciences (IFAS) is an Equal Opportunity Institution authorized to provide research, educational information and other services

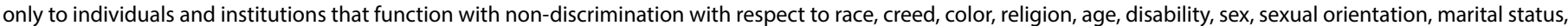

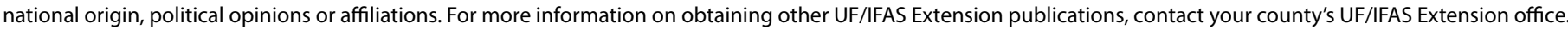
U.S. Department of Agriculture, UF/IFAS Extension Service, University of Florida, IFAS, Florida A \& M University Cooperative Extension Program, and Boards of County Commissioners Cooperating. Nick T. Place, dean for UF/IFAS Extension. 
to the balance of inflammatory and anti-inflammatory fats. Inflammation is a normal process for tissue healing; however, excessive or prolonged inflammation can damage skin and lead to dermatitis (Innes et al. 2018). Levels of essential fats, which have been found to provide benefits to skin's health and appearance (Oregon State University Linus Pauling Institute n.d.), were increased in the membranes of skin cells after hempseed oil supplementation (Callaway et al. 2005). Both skin dryness and itchiness improved after hempseed oil intervention (Callaway et al. 2005). However, this study was done with hemp oil; research is needed to test the effects of consuming hemp milk on inflammation and skin health.

Hemp seeds are being studied in relation to cardiovascular disease. Hemp seed protein has higher levels of arginine (an amino acid) (Rodriguez-Leyva et al. 2010), which, once consumed, changes into nitric oxide, a substance that relaxes blood vessels and improves circulation (Wells et al. 2005). Arginine has been associated with lower levels of C-reactive protein (a marker of inflammation), which is associated with heart disease and chronic inflammation (Wells et al. 2005). However, it is important to keep in mind that hemp milk contains low amounts of hemp seed per serving, so the level of arginine in hemp milk is low and thus might not have any protective effect against cardiovascular disease.

One benefit of hemp milk is that it is appropriate for allergies or intolerances to cow's milk, because it does not contain any milk proteins or the milk sugar lactose. Therefore, individuals allergic to cow's milk proteins, or with some degree of lactose intolerance, may safely consume hemp milk. Hemp is not one of the eight major food allergens (FDA n.d.), an advantage over almond and soy milk. However, hemp milk is not nutritionally equivalent to cow's milk.

Hemp milk is a source of unsaturated fats. The Dietary Guidelines for Americans recommends replacing saturated with unsaturated fats because it may reduce the risk of cardiovascular disease (USDA 2015).

\section{What are the possible risks of hemp milk?}

A disadvantage of hemp milk is its low level of protein per serving. Therefore, individuals must be aware that, if they choose to consume hemp milk instead of cow's milk, they should obtain adequate protein from other sources or enhance the protein content of hemp milk by adding a protein powder, such as pea or soy protein.

\section{Is hemp milk an appropriate choice for children?}

Adequate protein intake is especially important for children and adolescents. Child growth is negatively associated by the replacement of cow's milk with plant-based milk alternatives, possibly due to lower protein intakes (Morency et al. 2017). Children older than 2 years of age who do not consume cow's milk are encouraged to drink fortified plantbased milks with at least $6 \mathrm{~g}$ of protein per serving (Sethi et al. 2016), which exempts hemp milk. In addition, some hemp milks on the market are not fortified with vitamins A or D and thus are not comparable to some other plantbased milks or cow's milk as sources of these vitamins. Additionally, the form of calcium used to fortify hemp milk may have lower bioavailability than that provided in cow's milk (Bridges 2018, Singhal et al. 2017).

\section{Summary}

There may be health benefits of hempseed oil, but it is not known if hemp milk provides any health benefits. As with some other plant-based milks, hemp milk, although lower in calories and a source of healthy fat, is much lower in protein than cow's milk. Hemp milk should not be a complete beverage replacement for cow's milk in children and may not be an appropriate choice for older adults who have higher protein needs.

\section{References}

Bridges, Meagan. 2018. "Moo-Ove over, Cow's Milk: The Rise of Plant-Based Dairy Alternatives." Practical Gastroenterology 21. Accessed September 30, 2020. https://practicalgastro.com/wp-content/uploads/2019/07/Moo-ove-OverCow-Milk-Rise-of-Plant-Based-Dairy-Alternatives.pdf

Callaway, James, Ursula Schwab, Ilkka Harvima, Pirjo Halonen, Otto Mykkänen, Pekka Hyvönen, and Tomi Järvinen. 2005. "Efficacy of Dietary Hempseed Oil in Patients with Atopic Dermatitis." Journal of Dermatological Treatment 16 (2): 87-94. https://doi.org/10.1080/09546630510035832

Casula, Manuela, Elena Olmastroni, Marta Gazzotti, Federica Galimberti, Alberto Zambon, and Alberico L. Catapano. 2020. "Omega-3 Polyunsaturated Fatty Acids Supplementation and Cardiovascular Outcomes: Do Formulation, Dosage, and Baseline Cardiovascular Risk Matter? An Updated Meta-analysis of Randomized Controlled Trials." Pharmacological Research 160:105060. https://doi.org/10.1016/j.phrs.2020.105060 
Food and Drug Administration (FDA). n.d. "Food Allergies: What You Need to Know." Accessed September 30, 2020. https://www.fda.gov/Food/ResourcesForYou/ Consumers/ucm079311.htm

House, James, Jason Neufeld, and Gero Leson. 2010. "Evaluating the Quality of Protein from Hemp Seed (Cannabis sativa L.) Products through the Use of the Protein Digestibility-Corrected Amino Acid Score Method." Journal of Agricultural and Food Chemistry 58 (22): 11801-7. https://doi.org/10.1021/jf102636b

Innes, Jacqueline K., and Philip C. Calder. 2018. "Omega-6 Fatty Acids and Inflammation." Prostaglandins Leukot Essent Fatty Acids 132:41-48. https://doi.org/10.1016/j. plefa.2018.03.004.

Mitchell, Pat R., and Khalid M. Shammet. 2008. Hemp Food Product Base and Processes. Google Patents.

Morency, Marie-Elssa, Catherine S. Birken, Gerald Lebovic, Yang Chen, Mary L'Abbé, Grace J. Lee, Jonathon L. Maguire, and the TARGet Kids! Collaboration. 2017. "Association between Noncow Milk Beverage Consumption and Childhood Height." The American Journal of Clinical Nutrition 106 (2): 597-602. https://doi.org/10.3945/ajcn.117.156877

Oregon State University Linus Pauling Institute. n.d. "Essential Fatty Acids and Skin Health." Accessed September 30, 2020. https://lpi.oregonstate.edu/mic/health-disease/ skin-health/essential-fatty-acids\#overview.

Pacific Foods. n.d.-a. "Hemp Vanilla." Accessed September 30, 2020. https://www.pacificfoods.com/our-products/ hemp-plant-based-beverages/hemp-vanilla/

Pacific Foods. n.d.-b. "Unsweetened Hemp Original." Accessed September 30, 2020. https://www.pacificfoods. com/our-products/hemp-plant-based-beverages/ unsweetened-hemp-original/

Rodriguez-Leyva, Delfin, and Grant N. Pierce. 2010. "The Cardiac and Haemostatic Effects of Dietary Hempseed." Nutrition \& Metabolism 7 (1): 32. https://doi. org/10.1186/1743-7075-7-32

Sethi, Swati, S. K. Tyagi, and Rahul K. Anurag. 2016. "Plant-Based Milk Alternatives an Emerging Segment of Functional Beverages: A Review." Journal of Food Science and Technology 53 (9): 3408-3423. https://doi.org/10.1007/ s13197-016-2328-3
Singhal, Sarita, Robert D. Baker, and Susan S. Baker. 2017. "A Comparison of the Nutritional Value of Cow's Milk and Nondairy Beverages." Journal of Pediatric Gastroenterology and Nutrition 64 (5): 799-805. https://doi.org/10.1097/ mpg.0000000000001380

United States Department of Agriculture (USDA). 2015. "Dietary Guidelines for Americans 2015-2020." Accessed September 30, 2020. http://health.gov/ dietaryguidelines/2015/guidelines/

United States Department of Agriculture (USDA). n.d. Fooddata Central. Accessed September 30, 2020. https://fdc. nal.usda.gov/index.html

Wang, Qingling, Jiang Jiang, and Youling L. Xiong. 2018. "High Pressure Homogenization Combined with Ph Shift Treatment: A Process to Produce Physically and Oxidatively Stable Hemp Milk." Food Research International 106:487-494. https://doi.org/10.1016/j.foodres.2018.01.021

Wells, Brian J., Arch G. Mainous, and Charles J. Everett. 2005. "Association between Dietary Arginine and CReactive Protein." Nutrition 21 (2): 125-130. https://doi. org/10.1016/j.nut.2004.03.021 
Table 1. Nutrient profile of hemp milk compared to fat-free, low-fat, and whole cow's milk.

\begin{tabular}{|c|c|c|c|c|}
\hline & $\begin{array}{l}\text { Unsweetened hemp milk } \\
\text { ( } 1 \text { cup) }\end{array}$ & $\begin{array}{l}\text { Fat-free (skim) milk } \\
\text { ( } 1 \text { cup) }\end{array}$ & $\begin{array}{l}\text { Low-fat ( } 1 \%) \text { milk } \\
\text { ( } 1 \text { cup) }\end{array}$ & $\begin{array}{l}\text { Whole milk } \\
\text { ( } 1 \text { cup) }\end{array}$ \\
\hline Energy (calories) & 60 & 83 & 102 & 150 \\
\hline Protein (g) & 3 & 8 & 8 & 8 \\
\hline Total fat (g) & 4.5 & 0 & 2.4 & 8 \\
\hline Saturated fat (g) & NR & 0.1 & 1.5 & 4.5 \\
\hline Carbohydrate (g) & 0 & 12 & 12 & 12 \\
\hline Fiber (g) & 0 & 0 & 0 & 0 \\
\hline Total sugars (g) & 0 & 12 & 13 & 12 \\
\hline Vitamin A (mcg) & NR & 149 & 142 & 112 \\
\hline Vitamin B12 (mcg) & NR & 1 & 1 & 1 \\
\hline Vitamin D (mcg) & 2 & 3 & 3 & 3 \\
\hline Calcium (mg) & 257 & 298 & 305 & 276 \\
\hline Sodium (mg) & 110 & 102 & 107 & 105 \\
\hline Potassium (mg) & 100 & 381 & 366 & 322 \\
\hline
\end{tabular}

